\title{
Article \\ Data-Driven Modeling of Low Frequency Noise Using Capture-Emission Energy Maps
}

\author{
Jonghwan Lee
}

Citation: Lee, J. Data-Driven Modeling of Low Frequency Noise Using Capture-Emission Energy Maps. Appl. Sci. 2021, 11, 356. https://doi.org/10.3390/app11010356

Received: 13 November 2020 Accepted: 29 December 2020 Published: 31 December 2020

Publisher's Note: MDPI stays neutral with regard to jurisdictional clai$\mathrm{ms}$ in published maps and institutional affiliations.

Copyright: () 2020 by the author. Licensee MDPI, Basel, Switzerland. This article is an open access article distributed under the terms and conditions of the Creative Commons Attribution (CC BY) license (https:// creativecommons.org/licenses/by/ $4.0 /)$.
Department of System Semiconductor Engineering, Sangmyung University, Cheonan 31066, Korea; jhlee77@smu.ac.kr

Abstract: A new approach for modeling low frequency noise is presented to enable the predictions of noise behavior from negative bias temperature instability (NBTI). The noise model is based on a capture-emission energy (CEE) map describing the probability density function of widely distributed defect capture-emission activation energies. To enlarge the capture-emission energy window and to perform the accurate estimation of the recoverable component of CEE, the Gaussian mixture model (GMM) is applied to the CEE map. This approach provides an efficient identification of noise sources and an in-depth noise analysis under both stationary and cyclo-stationary conditions.

Keywords: low frequency noise; negative bias temperature instability; capture-emission energy map; gaussian mixture model

\section{Introduction}

In nanoscale CMOS devices, the stochastic behavior of a few defects can have a significant impact on the reliability of devices and their circuits [1-8]. The negative bias temperature instability (NBTI) is among the most critical reliability issues and can be described by capture-emission energy (CEE) maps [3-5,9-15]. Accurately modeling the NBTI relies on a combined methodology comprising thorough CEE maps and efficient models. However, CEE maps are limited by the stress-recovery measurement window [9,10,14-17]. An accurate estimation of the parameters of CEE distribution is crucial to enable full lifetime calculation of NBTI without measurement time extrapolation. The kinetics of defects responsible for NBTI has been explained by the random capture-emission process of charge carriers caused by oxide traps [9-15,18,19]. Moreover, it has been found that the recoverable component of NBTI and random telegraph signal (RTS) noise are due to same defects [19-25]. The discrete fluctuations of RTSs with widely distributed time or energy scales are the main source of low frequency $(1 / f)$ noise in nanoscale MOSFETs [20,21,26-29]. For the statistical analysis and rigorous modeling of noise behavior, it is crucial to incorporate the distribution of defects based on CEE maps, including large signal AC operations [27-30]. In particular, since the static random-access memory (SRAM) cell is a highly scaled device, the threshold voltage variation due to RTS is a major source of degradation in SRAM characteristics [30]. In the CEE map framework, the time-dependent variability of degradation such as NBTI and noise is reproduced under different bias and duty cycle conditions. In this study, CEE-based noise models are newly presented to allow a quantitative prediction for RTS and $1 / f$ noise. The models utilize a Gaussian mixture model (GMM) for enlarging the capture-emission energy window and clustering the recoverable component from the fitted CEE map.

\section{Theoretical Model}

The capture-emission process of charge carriers is consistent with non-radiative multiphonon theory based on linear electron-phonon coupling [1]. In this theory, the captureemission times are expressed as $\tau_{c, e}\left(E_{c}\right)=\tau_{0} \exp \left(E_{c, e} / k_{b} T\right)$ where $\tau_{0}$ is the temperatureindependent pre-factor, $k_{b}$ is the Boltzmann constant, $T$ is the temperature, and $E_{c}$ and $E_{e}$ 
are the capture and emission activation energy, respectively. The NBTI-induced threshold voltage shifts $\Delta V_{t h}$ is related to the capture-emission processes of individual defects with a broad distribution of characteristic times. The step height observed in $\Delta V_{t h}$ for small-area devices is due to individual defects, while for large-area devices similar defects are grouped together using a suitable distribution of defects $[1,19]$. This distribution is visualized in the CEE map, which can be directly obtained from experimental recovery curves of $\Delta V_{t h}$ for various stress conditions by using $D\left(\tau_{c}, \tau_{e}\right)=-\partial^{2} \Delta V_{t h}\left(\tau_{c}, \tau_{e}\right) / \partial \tau_{c} \partial \tau_{e}$ [1]. The defect density map is represented in agreement with bivariate Gaussian distribution, describing the permanent and recoverable components. In the activation energy space, the density $D\left(E_{c}, E_{e}\right)$ is constructed by the joint probability density function $[9,10,14,15]$

$$
D\left(E_{c}, E_{e}\right)=\frac{D_{0}}{2 \pi \sigma_{c} \sigma_{e} \sqrt{1-\rho^{2}}} e^{\left[-\frac{1}{2\left(1-\rho^{2}\right)}\left(\frac{\left(E_{\mathcal{C}}-\mu_{\mathcal{C}}\right)^{2}}{\sigma_{c}^{2}}-\frac{2 \rho\left(E_{\mathcal{C}}-\mu_{\mathcal{C}}\right)\left(E_{\mathcal{e}}-\mu_{\mathcal{e}}\right)}{\sigma_{\mathcal{C}} \sigma_{e}}+\frac{\left(E_{\mathcal{e}}-\mu_{\mathcal{e}}\right)^{2}}{\sigma_{e}^{2}}\right)\right]}
$$

where $D_{0}=\left(V_{s} / V_{0}\right)^{\gamma}$ is a voltage-dependent amplitude with the stress voltage $V_{s}$ and the constants $V_{0}$ and $\gamma \cdot \mu_{c, e}$ and $\sigma_{c, e}$ are the mean values and standard deviations of $E_{c, e}$, respectively. A correlation coefficient is defined as $\rho=\operatorname{covariance}\left(E_{c}, E_{e}\right) / \sigma_{c} \sigma_{e}$. For defects being charged up to the stress time $t_{s}$ and not yet being discharged at the recovery time $t_{r}$, the threshold voltage shift is calculated by integrating over the activation energy map as follows $[9,10,14,15]$

$$
\begin{aligned}
\Delta V_{t h}\left(t_{s}, t_{r}\right) & =\int_{0}^{\infty} \int_{0}^{\infty} D_{r}\left(E_{c}, E_{e}\right) P_{o c c}\left(E_{c}, E_{e}, t_{s}, t_{r}\right) d E_{c} d E_{e} \\
& +\int_{0}^{\infty} \int_{0}^{\infty} D_{p}\left(E_{c}, E_{e}\right) P_{o c c}\left(E_{c}, E_{e}, t_{s}, t_{r}\right) d E_{c} d E_{e}
\end{aligned}
$$

where $D_{r}\left(E_{c}, E_{e}\right)$ and $D_{p}\left(E_{c}, E_{e}\right)$ are the recoverable and permanent component, respectively, and $P_{\text {occ }}\left(E_{c}, E_{e}, t_{s}, t_{r}\right)$ is the occupancy probability map for the applied stress and recovery waveform. The occupancy probability $P_{o c c}$ after $t_{s}$ and $t_{r}$ is described by [1]

$$
P_{o c c}\left(E_{c}, E_{e}, t_{s}, t_{r}\right)=\left(1-e^{-\frac{t_{S}}{\tau_{c}\left(E_{\mathcal{C}}\right)}}\right) e^{-\frac{t_{r}}{\tau_{e}\left(E_{e}\right)}}
$$

For AC stress and recovery patterns with the signal duty cycle $\alpha$, and the signal frequency $f$, the probability to be occupied after the high gate-source voltage $V_{g s}^{h}$ will be written as [30]

$$
P_{o c c}\left(E_{c}, E_{e}, t_{s}, t_{r}\right)=\frac{1-e^{-\frac{\alpha}{f \tau_{\mathcal{C}}}}}{1-e^{-\frac{1}{f}\left(\frac{\alpha}{\tau_{\mathcal{C}}}+\frac{1-\alpha}{\tau_{e}}\right)}}\left(1-e^{-t_{s}\left(\frac{\alpha}{\tau_{\mathcal{C}}}+\frac{1-\alpha}{\tau_{e}}\right)}\right)
$$

Trap-related processes are the most likely cause of the fluctuation in the number of charge carriers. The contribution of multiple-trap levels leads to a noise power spectrum expressed by the superposition of a single Lorentzian noise as follows [27]

$$
S_{N}(\omega)=\sum_{i=1}^{N_{t}} S_{N, i}(\omega)=4 \sum_{i=1}^{N_{t}} \Delta N_{i}^{2} \frac{\tau_{e f f, i}}{1+\omega^{2} \tau_{e f f, i}^{2}}
$$

where $\Delta N_{i}^{2}=A_{i}^{2} \tau_{c, i} \tau_{e, i} /\left(\tau_{c, i}+\tau_{e, i}\right)^{2}$ is the variance of the fluctuation $\Delta N$ in the number of carriers $N, A_{i}$ is the amplitude, $N_{t}$ is the number of traps following a Poisson distribution, and $\omega$ is the angular frequency. With the assistance of capture-emission activation energies, a change in charge state can occur even if the energy levels of the traps are higher or lower than the Fermi level compared to a few $k_{b} T$, resulting in $1 / f$ noise [31-35]. Assuming that $A_{i}$ is random variables identically distributed and the effective times constants are 
statistically independent, the noise power spectrum of the occupancy $P_{o c c}\left(E_{c}, E_{e}, t_{s}, t_{r}\right)$ of the traps in the energy element $\Delta E_{c}$ and $\Delta E_{e}$ is given by [34,35]

$$
S_{N}(\omega)=4 N_{t}^{*} A^{2} \frac{\tau_{c} \tau_{e}}{\left(\tau_{c}+\tau_{e}\right)^{2}} \frac{\tau_{e f f, i}}{1+\omega^{2} \tau_{e f f, i}^{2}} P_{o c c}\left(E_{c}, E_{e}, t_{s}, t_{r}\right) \Delta E_{c} \Delta E_{e}
$$

where $N_{t}^{*}$ is the trap density per unit area. Given that the trap distribution is described by the normalized density $D_{r}^{*}\left(E_{c}, E_{e}\right)$ of the recoverable component, the average noise power spectrum for a device with a width $W$ and length $L$ is converted to an integral expression

$$
\overline{S_{N}(\omega)}=4 N_{t}^{*} W L A^{2} \int_{0}^{\infty} \int_{0}^{\infty} \frac{\tau_{c} \tau_{e}}{\left(\tau_{c}+\tau_{e}\right)^{2}} \frac{\tau_{e f f}}{1+\omega^{2} \tau_{e f f}^{2}} D_{r}^{*}\left(E_{c}, E_{e}\right) P_{o c c}\left(E_{c}, E_{e}, t_{s}, t_{r}\right) d E_{c} d E_{e}
$$

where $\tau_{e f f}=\left(1 / \tau_{c}+1 / \tau_{e}\right)^{-1}$ is the effective time. Note that the term $P_{o c c}\left(E_{c}, E_{e}\right)$ is similar to the occupancy of traps $f_{T}\left(1-f_{T}\right)$ described by a Fermi function $f_{T}$, reflecting the fact that traps with higher occupancy probability contribute to noise power. The equivalent gate voltage noise power spectrum of MOSFETs in the linear region is given by $S_{V_{g}}(\omega)=q^{2} \overline{S_{N}(\omega)} /\left(W^{2} L^{2} C_{o x}^{2}\right)$ where $C_{o x}$ is the gate oxide capacitance per unit area. Under cyclo-stationary square wave excitation, the noise behavior is governed by the time during the on-state, $\tau_{c, o n}$ and $\tau_{e, o n}$, and the times during the off-state, $\tau_{c, o f f}$ and $\tau_{e, o f f}$. The effective and characteristic times are written as [28]

$$
\tau_{e f f}^{*}=\left(\frac{\alpha}{\tau_{o n}}+\frac{1-\alpha}{\tau_{o f f}}\right)^{-1}, \tau_{c / e}^{*}=\left(\frac{\alpha}{\tau_{c / e, o n}}+\frac{1-\alpha}{\tau_{c / e, o f f}}\right)^{-1}
$$

with $\tau_{o n}=\left(1 / \tau_{c, o n}+1 / \tau_{e, o n}\right)^{-1}, \tau_{o f f}=\left(1 / \tau_{c, o f f}+1 / \tau_{e, o f f}\right)^{-1}$.

For an accurate estimation of CEE distribution, a GMM of the distributions appears to be a natural choice for two components of Gaussian distributions. Given a two-dimensional random variable $E_{a}=\left(E_{c}, E_{e}\right)$, with mean $\mu_{i}$ and covariance $\Sigma_{i}$, the GM density function of two Gaussian components is given by [36-39]

$$
p\left(E_{a}\right)=\sum_{i=1}^{2} \pi_{i} \mathcal{N}\left(E_{a} \mid \mu_{i}, \Sigma_{i}\right)
$$

where $\pi_{i}$ is the weight of the $i$-th mixture component, subject to $0 \leq \pi_{i} \leq 1$ and $\pi_{1}+\pi_{2}=1$. The $i$-th independent normal distribution is

$$
N\left(E_{a} \mid \mu_{i}, \Sigma_{i}\right)=\frac{1}{\sqrt{2 \pi\left|\Sigma_{i}\right|}} e^{-\frac{1}{2}\left(E_{a}-\mu_{i}\right)^{T} \Sigma_{i}^{-1}\left(E_{a}-\mu_{i}\right)}
$$

The expectation maximization (EM) algorithm is used to compute the parameters of each Gaussian component [36-38]. It is an iterative algorithm that estimates a maximum likelihood solution of the parameters, based on an expectation (E) step and a maximization (M) step. The GMM is used for clustering Gaussian components, by realizing that two bivariate Gaussian distributions of fitted CEE map can represent clusters. The soft clustering method is applied to estimate cluster membership posterior probabilities [39]. The method assigns a cluster membership score to each data point and ranks the points by their cluster membership score, allowing some data points to belong to multiple clusters.

\section{Results and Discussion}

The devices used for measurements were fabricated using a manufacturable remote plasma nitride oxide (RPNO) process. The gate oxide of devices used for measurements was grown with both furnace and rapid-thermal based processes. Following $\mathrm{N}_{2}$-plasma nitridation and post-nitridation anneal of the gate oxide, an undoped poly-Si gate layer was deposited. For p+ gates, B was implanted, and rapid-thermal annealing was then 
performed in $\mathrm{N}_{2}$ for different durations. The threshold voltage shifts are obtained on pMOSFETs $\left(W=10 \mu \mathrm{m}, L=0.14 \mu \mathrm{m}, t_{o x}=2.2 \mathrm{~nm}\right)$, performing the ultrafast measure-stressmeasurements $[9,10]$. With a measuring delay time of $1 \mu \mathrm{s}$, the temperature-accelerated measurement scheme was used to extend to the experimental stress-recovery window of $10^{7} \mu \mathrm{s}$. Noise measurements are conducted using an HP 3582A spectrum analyzer $(1 \mathrm{~Hz}-25 \mathrm{kHz})$ and a Brookdeal 5004 low noise voltage amplifier connected at the output terminal. A bias resistance at the output terminal is chosen to be 10 times larger than the small signal channel resistance. The noise power spectrum is adjusted to subtract the noise due to the measurement system, which consists of the noise due to the amplifier and the bias resistance, and the thermal noise of device under test. Figure 1a shows experimental recovery data of $\Delta V_{t h}$ following stress time with numerical data for $V_{g s}=-2.0 \mathrm{~V}$ and $T=125^{\circ} \mathrm{C}$. Numerical data of $\Delta V_{t h}$ are obtained by integrating the CEE distribution in (2), which is calculated by taking the derivatives of measured data, as shown in Figure $1 \mathrm{~b}$. Figure 2a shows a scatter plot of GMM data using the soft clustering method. The cluster membership posterior probabilities in the interval $(0.45,0.55)$ are members of both components with six shared data points. The estimated parameters of permanent component are $\mu_{c 1}=1.593 \mathrm{eV}, \mu_{e 1}=1.604 \mathrm{eV}, \sigma_{c 1}=0.203 \mathrm{eV}, \sigma_{e 1}=0.098 \mathrm{eV}, \rho_{1}=0.035$, and those of recoverable component are $\mu_{c 2}=0.908 \mathrm{eV}, \mu_{e 2}=0.997 \mathrm{eV}, \sigma_{c 2}=0.376 \mathrm{eV}, \sigma_{e 2}=0.317 \mathrm{eV}$, $\rho_{2}=0.8537$. Using the estimated parameters of GMM model, the CEE distributions are produced in the extended energy space exceeding experimental window, as observed in Figure $2 b$. The inset of Figure $2 b$ shows a plot of the relative error values which are less than $0.5 \%$.

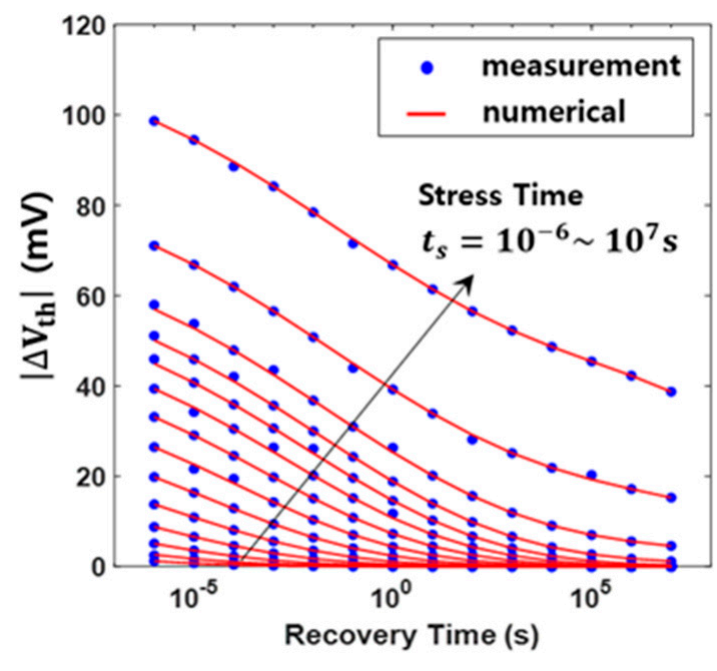

(a)

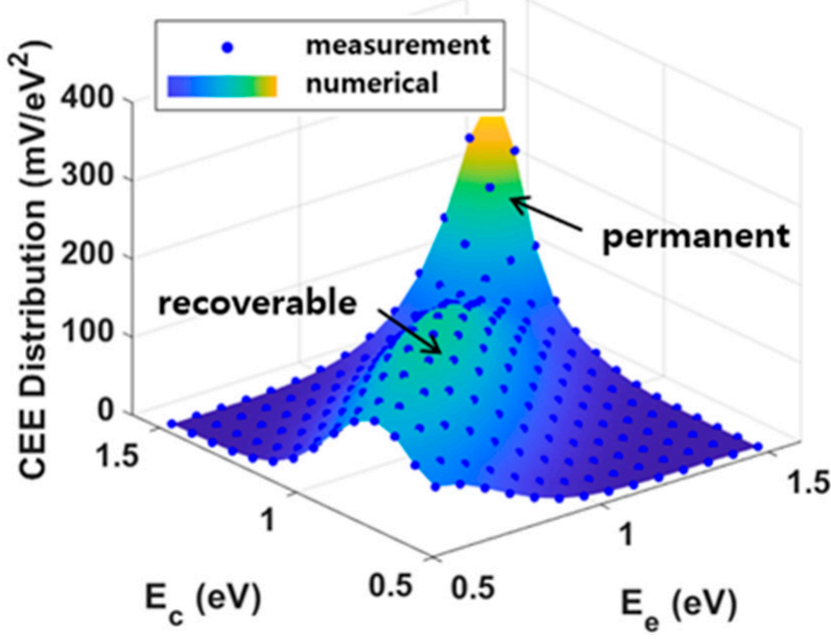

(b)

Figure 1. Threshold voltage shifts $\Delta V_{t h}$ and capture-emission energy (CEE) map. (a) Numerical data with measured data of $\Delta V_{t h}$. (b) Comparison of numerical data with measured data of CEE map.

To investigate how the trap distribution influences RTS noise, the integrand of $\overline{S_{N}(\omega)}$ for the recoverable component in (7) is numerically calculated and visualized as a function of $E_{e}$ and $E_{c}$ under stationary DC condition at a particular frequency. It is shown in Figure 3a that a peak is distinct at $E_{c}=1.10 \mathrm{eV}$ and $E_{e}=0.65 \mathrm{eV}$ for $f=1 \mathrm{~Hz}$, and moves to relatively low activation energies $E_{c}=0.75 \mathrm{eV}$ and $E_{e}=0.45 \mathrm{eV}$ as the frequency increases to $f=1 \mathrm{kHz}$. As illustrated in the inset of Figure 3a, these results arise from different RTSs, indicating that faster components of shallow trap states for $f=1 \mathrm{kHz}$ are situated close to lower activation energies [40]. To examine the behavior of cyclo-stationary RTS, the times are modeled as $\tau_{c, o f f}=m \cdot \tau_{c, o n}$ and $\tau_{e, o f f}=\tau_{e, o n} / m$, where the factor $m$ is assumed to be greater than one $[28,40]$. In the off state, $\tau_{c}$ and $\tau_{e}$ are multiplied and divided by $m$, as they increase and decrease with a decrease in the gate voltage, respectively [28,40]. For $m=50$, a peak moves to higher emission energy $E_{e}$, as shown in Figure $3 \mathrm{~b}$. 


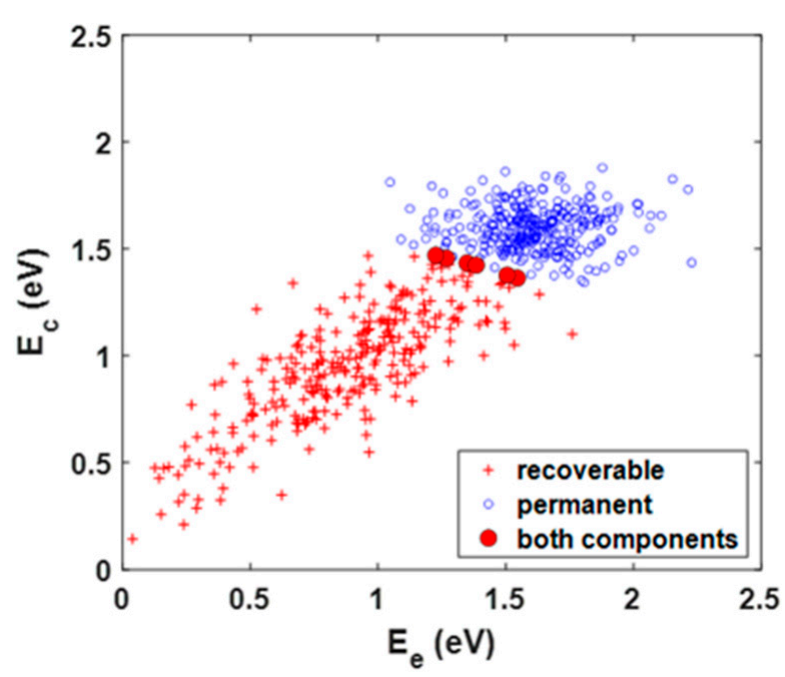

(a)

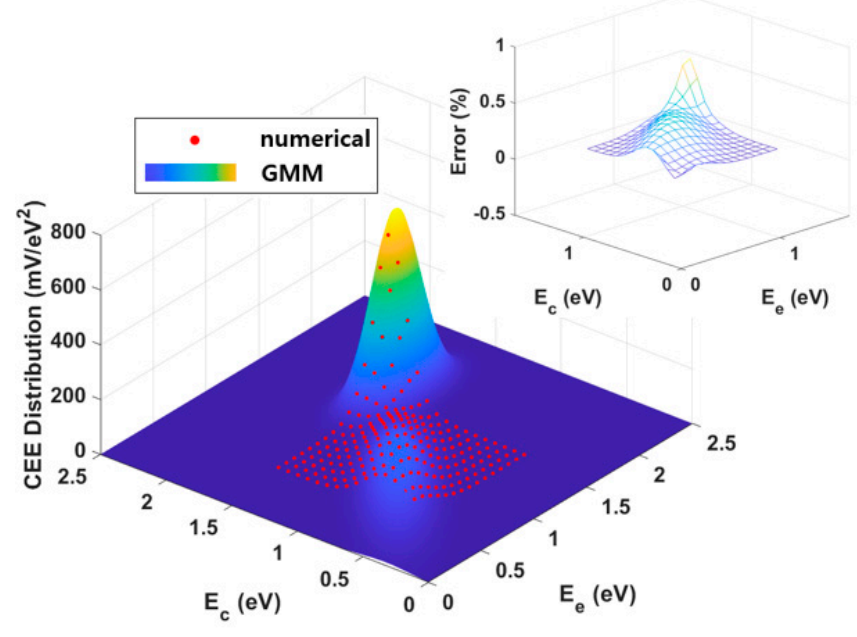

(b)

Figure 2. CEE map with fitted Gaussian mixture (GMM) using soft clustering. (a) Scatter plot of GMM data. (b) Comparison of GMM data with numerical data of CEE map.

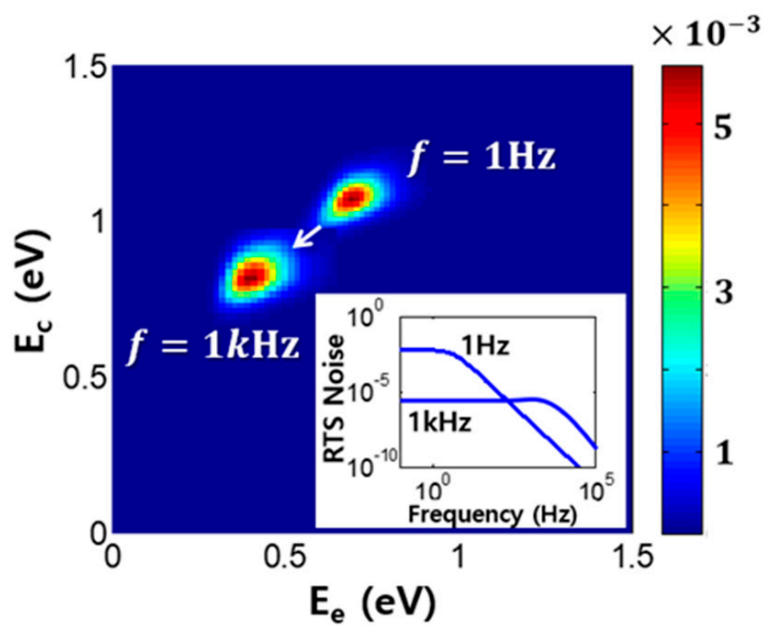

(a)

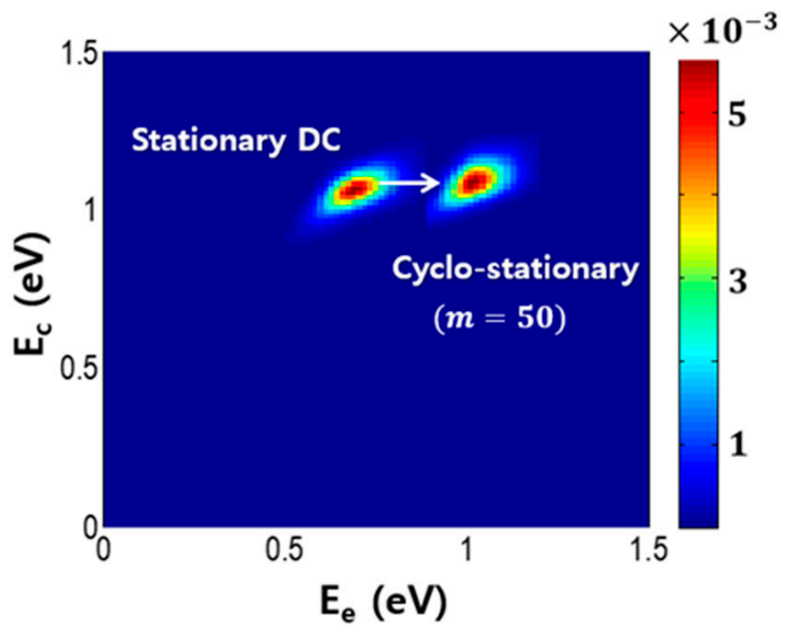

(b)

Figure 3. Integrand of $\overline{S_{N}(\omega)}$ for the recoverable component. (a) $f=1 \mathrm{~Hz}$ and $f=1 \mathrm{kHz}$ under stationary condition for $V_{g s}=-2 \mathrm{~V}$. (b) $f=1 \mathrm{~Hz}$ and $m=50$ under cyclo-stationary condition for $V_{g s}=-2 \mathrm{~V}$.

$1 / f$ noise power is calculated by using (7) with the recoverable component obtained from GMM with soft clustering. It is observed in Figure 4a that the recoverable component of CEE map gives a $1 / f$ spectrum form with a slope of -1 . For asymmetric RTSs $\left(\tau_{c, \text { on }} / \tau_{e, o n}\right)$, cyclo-stationary condition may lead to an increase in noise power [28]. Figure $4 \mathrm{~b}$ shows a linear relationship between $S_{V_{g}}(\omega)$ and $\Delta V_{t h}$ after a stress time $t_{s}=1000 \mathrm{~s}$. The same strong correlation is also observed for the device with $\mathrm{SiON} /$ poly-Si gate [20]. 


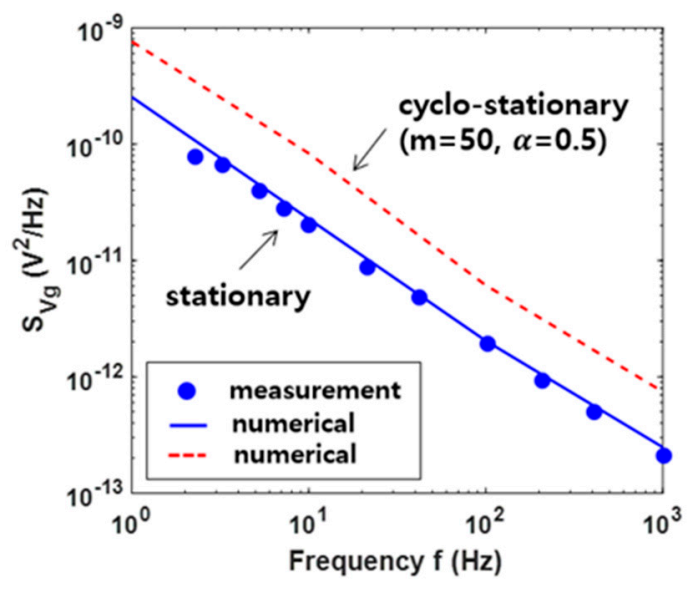

(a)

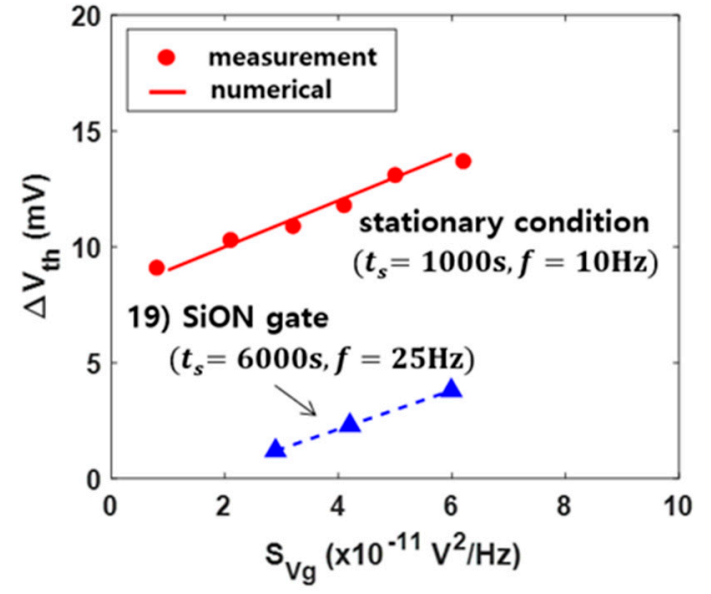

(b)

Figure 4. $1 / f$ noise spectrum and threshold voltage shifts for $t_{s}=1000 \mathrm{~s}$. (a) $S_{V_{g}}(\omega)$ for $V_{g s}=-2 \mathrm{~V}, N_{t}^{*}=2.0 \times 10^{12} \mathrm{~cm}^{-2}$ (b) $S_{V_{g}}(\omega)$ versus $\Delta V_{t h}$ for $N_{t}^{*}=2.0 \times 10^{12} \mathrm{~cm}^{-2}$.

\section{Conclusions}

In conclusion, with a recoverable component of NBTI stress-recovery, a new noise model is formulated based on a fitted CEE map with soft clustering of GMM. The proposed model offers the following unique features: (1) The model is based on the occupancy of defects in the capture-emission energy map. Therefore, it allows modeling of the noise under both stationary as well as cyclo-stationary excitation conditions. (2) The GMM with soft clustering enlarges the CEE window and performs independent estimations of two distinct components of CEE map, enabling the accurate calculation of noise characteristics. (3) New theoretical expressions can capture the noise behavior in terms of activation energies and provide the identification of noise sources and the reliable prediction of $1 / f$ noise from NBTI.

Funding: This work was supported by the National Research Foundation of Korea (NRF) grant funded by the Korea government (MSIT) (No. 2019R1F1A1050640).

Data Availability Statement: No new data were created or analyzed in this study. Data sharing is not applicable to this article.

Conflicts of Interest: The author declares no conflict of interest.

\section{References}

1. Grasser, T. Stochastic charge trapping in oxides: From random telegraph noise to bias temperature instabilities. Microelectron. Reliab. 2012, 52, 39-70. [CrossRef]

2. Grasser, T.; Kaczer, B.; Goes, W.; Reisinger, H.; Aichinger, T.; Hehenberger, P.; Wagner, P.-J.; Schanovsky, F.; Franco, J.; Luque, M.T.; et al. The Paradigm Shift in Understanding the Bias Temperature Instability: From Reaction-Diffusion to Switching Oxide Traps. IEEE Trans. Electron Devices 2011, 58, 3652-3666. [CrossRef]

3. Reisinger, H.; Grasser, T.; Gustin, W.; Schlünder, C. The statistical analysis of individual defects constituting NBTI and its implications for modeling DC- and AC-stress. In Proceedings of the 2010 IEEE International Reliability Physics Symposium, Anaheim, CA, USA, 2-6 May 2010; pp. 7-15.

4. Reisinger, H.; Grasser, T.; Schlünder, C. A study of NBTI by the statistical analysis of the properties of individual defects in pMOSFETs. In Proceedings of the 2009 IEEE International Integrated Reliability Workshop Final Report, South Lake Tahoe, CA, USA, 18-22 October 2009; pp. 30-35.

5. Reisinger, H.; Blank, O.; Heinrigs, W.; Gustin, W.; Schlünder, C. A comparison of very fast to very slow components in degradation and recovery due to NBTI and bulk hole trapping to existing physical models. IEEE Trans. Device Mater. Reliab. 2007, 7, 119-129. [CrossRef]

6. Reisinger, H.; Grasser, T.; Ermisch, K.; Nielen, H.; Gustin, W.; Schlünder, C. Understanding and modeling AC BTI. In Proceedings of the 2011 IEEE International Reliability Physics Symposium, Monterey, CA, USA, 10-14 April 2011; pp. 597-604. 
7. Bucolo, M.; Buscarino, A.; Famoso, C.; Fortuna, L.; Frasca, M. Control of imperfect dynamical systems. Nonlinear Dyn. 2019, 98, 2989-2999. [CrossRef]

8. Zhao, K.; Stathis, J.H.; Linder, B.P.; Cartier, E.; Kerber, A. PBTI under dynamic stress: From a single defect point of view. In Proceedings of the 2011 IEEE International Reliability Physics Symposium, Monterey, CA, USA, 10-14 April 2011 ; pp. 372-380.

9. Puschkarsky, K.; Reisinger, H.; Schlünder, C.; Gustin, W.; Grasser, T. Voltage-dependent activation energy maps for analytic lifetime modeling of NBTI without time extrapolation. IEEE Trans. Electron Devices 2018, 65, 4764-4771. [CrossRef]

10. Puschkarsky, K.; Reisinger, H.; Rott, G.A.; Schlünder, C.; Gustin, W.; Grasser, T. An efficient analog compact NBTI model for stress and recovery based on activation energy maps. IEEE Trans. Electron Devices 2019, 66, 4623-4630. [CrossRef]

11. Giering, K.U.; Puschkarsky, K.; Reisinger, H.; Rzepa, G.; Rott, G.; Vollertsen, R.; Grasser, T.; Jancke, R. NBTI degradation and recovery in analog circuits: Accurate and efficient circuit-level modeling. IEEE Trans. Electron Devices 2019, 66, 1662-1668. [CrossRef]

12. Ma, C.; Mattausch, H.J.; Matsuzawa, K.; Yamaguchi, S.; Hoshida, T.; Imade, M.; Koh, R.; Arakawa, T.; Miura-Mattausch, M. Universal NBTI compact model for circuit aging simulation under any stress conditions. IEEE Trans. Device Mater. Reliab. 2014, 14, 818-825. [CrossRef]

13. Wang, W.; Yang, S.; Bhardwaj, S.; Vrudhula, S.; Liu, F.; Cao, Y. The impact of NBTI effect on combinational circuit: Modeling, simulation, and analysis. IEEE Trans. Very Large Scale Integr. VLSI Syst. 2009, 18, 173-183. [CrossRef]

14. Lagger, P.; Reiner, M.; Pogany, D.; Ostermaier, C. Comprehensive study of the complex dynamics of forward bias-induced threshold voltage drifts in GaN based MIS-MEMTs by stress/recovery experiments. IEEE Trans. Electron Devices 2014, 61, 1022-1030. [CrossRef]

15. Putcha, V.; Franco, J.; Vais, A.; Sioncke, S.; Kaczer, B.; Linten, D.; Groeseneken, G. On the apparent non-Arrhenius temperature dependence of charge trapping in IIIV/high-k MOS stack. IEEE Trans. Electron Devices 2018, 65, 3689-3696. [CrossRef]

16. Pobegen, G.; Grasser, T. On the distribution of NBTI time constants on a long, temperature-accelerated time scale. IEEE Trans. Electron Devices 2013, 60, 2148-2155. [CrossRef]

17. Pobegen, G.; Aichinger, T.; Nelhiegbel, M.; Grasser, T. Understanding temperature acceleration for NBTI. In Proceedings of the 2011 International Electron Device Meeting, Washington, DC, USA, 5-7 December 2011; pp. 614-617.

18. Tewksbury, T.L.; Lee, H.S. Characterization, modeling, and minimization of transient threshold voltage shifts in MOSFET's. IEEE J. Solid State Circuits 1994, 29, 239-252. [CrossRef]

19. Grasser, T.; Rott, K.; Reisinger, H.; Waltl, M.; Franco, J.; Kaczer, B. A unified perspective of RTN and BTI. In Proceedings of the 2014 IEEE International Reliability Physics Symposium, Waikoloa, HI, USA, 1-5 June 2014; pp. 4A.5.1-4A.5.7.

20. Kaczer, B.; Grasser, T.; Martin-Martinez, J.; Simoen, E.; Aoulaiche, M.; Roussel, P.J.; Groseneken, G. NBTI from the perspective of defect states with widely distributed time scales. In Proceedings of the 2009 IEEE International Reliability Physics Symposium, Montreal, QC, Canada, 26-30 April 2009; pp. 55-60.

21. Huard, V. Two independent components modeling for negative bias temperature instability. In Proceedings of the 2010 IEEE International Reliability Physics Symposium, Anaheim, CA, USA, 2-6 May 2010; pp. 33-42.

22. Tsukamoto, Y.; Toh, S.O.; Shin, C.; Mairena, A.; Liu, T.-J.K.; Nikolic, B. Analysis of the relationship between random telegraph signal and negative bias temperature instability. In Proceedings of the 2010 IEEE International Reliability Physics Symposium, Anaheim, CA, USA, 2-6 May 2010; pp. 1117-1121.

23. Bury, E.; Degraeve, R.; Cho, M.J.; Kaczer, B.; Goes, W.; Grasser, T.; Horiguchi, N.; Groeseneken, G. Study of (correlated) trap sites in SILC, BTI and RTN in SiON and HKMG devices. In Proceedings of the 21th International Symposium on the Physical and Failure Analysis of Integrated Circuits (IPFA), Marina Bay Sands, Singapore, 30 June-4 July 2014; pp. 250-253.

24. Both, T.H.; Furtado, G.F.; Wirth, G.I. Modeling and simulation of the charge trapping component of BTI and RTS. Microelectron. Reliab. 2018, 80, 278-283. [CrossRef]

25. Wirth, G.I.; da Silva, R.; Kaczer, B. Statistical model for MOSFET bias temperature instability component due to charge trapping. IEEE Trans. Electron Devices 2011, 58, 2743-2751. [CrossRef]

26. Grasser, T.; Reisinger, H.; Goes, W.; Aichinger, T.; Hehenberger, P.; Wagner, P.J.; Nelhiebel, M.; Franco, J.; Kaczer, B. Switching oxide traps as the missing link between negative bias temperature instability and random telegraph noise. In Proceedings of the 2009 International Electron Device Meeting, Baltimore, MD, USA, 7-9 December 2009; pp. 729-732.

27. Da Silva, R.; Wirth, G.; Brusamarello, L. An appropriate model for the noise power spectrum produced by traps at the $\mathrm{Si}_{-} \mathrm{SiO}{ }_{2}$ interface: A study of the influence of a time-dependent Fermi level. J. Stat. Mech. Theory Exp. 2008, 2008, P10015. [CrossRef]

28. Wirth, G.; da Silva, R. Low-frequency noise spectrum of cyclo-stationary random telegraph signals. Electr. Eng. 2008, 90, 435-441. [CrossRef]

29. Da Silva, R.; Wirth, G.I.; Brederlow, R. Novel analytical and numerical approach to modeling low-frequency noise in semiconductor devices. Phys. A Stat. Mech. Appl. 2006, 362, 277-288. [CrossRef]

30. Weckx, P.; Kaczer, B.; Toledano-Luque, M.; Grasser, T.; Roussel, P.J.; Kukner, H.; Raghavan, P.; Catthoor, F.; Groeseneken, G. Defect-based methodology for workload-dependent circuit lifetime projections-Application to SRAM. In Proceedings of the 2013 IEEE International Reliability Physics Symposium, Anaheim, CA, USA, 14-18 April 2013; pp. 3A.4.1-3A.4.7.

31. Fleetwood, D.M. $1 / f$ noise and defects in microelectronic materials and devices. IEEE Trans. Nucl. Sci. 2015, 62, 1462-1486. [CrossRef] 
32. Fleetwood, D.M.; Xiong, H.D.; Lu, Z.Y.; Nicklaw, C.J.; Felix, J.A.; Schrimpf, R.D.; Pantelides, S.T. Unified model of hole trapping, $1 / f$ noise, and thermally stimulated current in MOS devices. IEEE Trans. Nucl. Sci. 2002, 49, 2674-2683. [CrossRef]

33. Simoen, E.; Claeys, C. On the flicker noise in submicron silicon MOSFETs. Solid-State Electron. 1999, 43, 865-882. [CrossRef]

34. Wong, H.; Cheng, Y.C. Modeling of low-frequency noise in metal-oxide-semiconductor field-effect transistor with electron trapping-detrapping at oxide-silicon interface. IEEE Trans. Electron Devices 1991, 38, 1883-1888. [CrossRef]

35. Surya, C.; Hsiang, T.Y. A thermal activation model for $1 / f^{y}$ noise in Si-MOSFETs. Solid State Electron. 1988, 31, 959-964. [CrossRef]

36. Shimizu, H.; Awano, H.; Hiromoto, M.; Sato, T. Automation of model parameter estimation for random telegraph noise. IEICE Trans. Fundam. Electron. Commun. Comput. Sci. 2014, E97-A, 2383-2392. [CrossRef]

37. Singh, R.; Pal, B.C.; Jabr, R.A. Statistical representation of distribution system loads using Gaussian mixture model. IEEE Trans. Power Syst. 2010, 25, 29-37. [CrossRef]

38. Bui, D.T.; Hoang, N.D. A Bayesian framework based on a Gaussian mixture model and radial-basis-function Fisher discriminant analysis (BayGmmKda V1.1) for spatial prediction of floods. Geosci. Model Dev. 2017, 10, 3391-3409. [CrossRef]

39. Peters, G.; Crespo, F.; Lingras, P.; Weber, R. Soft clustering-Fuzzy and rough approaches and their extensions and derivatives. Int. J. Approx. Reason. 2013, 54, 307-322. [CrossRef]

40. van der Wel, A.P.; Klumperink, E.A.M.; Hoekstra, E.; Nauta, B. Relating random telegraph signal noise in metal-oxidesemiconductor transistors to interface trap energy distribution. Appl. Phys. Lett. 2005, 87, 183507. [CrossRef] 